\title{
Proposal for using optical transition radiation for electron beam alignment and emittance measurement for the free electron laser experiments at $\mathrm{ATF}^{*}$
}

\author{
Xu Z. Qiu, Xijie Wang and Ilan Ben-Zvi \\ Building $725 \mathrm{C}$ \\ National Synchrotron Light Source \\ Brookhaven National Laboratory \\ Upton, N.Y., 11973
}

\begin{abstract}
Optical trnasiton radiation (OTR) produced from thin intercepting foils have been employed to image the spatial profile of the electron beam in several free electron laser experiments. It was found that the images from OTR were significantly sharper than the images produced from phosphor screens. Furthermore, OTR's sensitivity of its angular distribution and polarization to energy and divergence of the electron beam was exploited to diagnose energy and emittance of the electron beam. OTR has been proven to be vital in electron beam alignment in FEL experiments. In this report, we will give a summary of the basic theory of transition radiation and techniques using transition radiation for electron beam imaging and emittance measurement. We will then explored the possibility for employing these techniques in the HGHG FEL and the visible FEL experiments in ATF.
\end{abstract}

*Work performed under the auspices of the U.S. Department of Energy

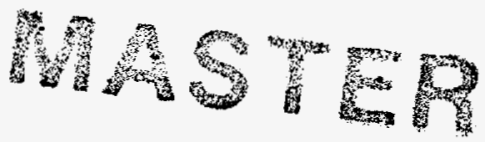




\section{DISCLAIMER}

This report was prepared as an account of work sponsored by an agency of the United States Government. Neither the United States Government nor any agency thereof, nor any of their employees, make any warranty, express or implied, or assumes any legal liability or responsibility for the accuracy, completeness, or usefulness of any information, apparatus, product, or process disclosed, or represents that its use would not infringe privately owned rights. Reference herein to any specific commercial product, process, or service by trade name, trademark, manufacturer, or otherwise does not necessarily constitute or imply its endorsement, recommendation, or favoring by the United States Government or any agency thereof. The views and opinions of authors expressed herein do not necessarily state or reflect those of the United States Government or any agency thereof. 


\section{DISCLAIMER}

Portions of this document may be illegible in electronic image products. Images are produced from the best available original document. 


\section{Introduction}

The earliest observation of optical transition radiation (OTR) were those made in connection with studies of $x$-ray tubes at the turn of the century. The cause of the light, emitted by electrons impinging on the anodes of the tubes, was not at first understood. In 1919 Lilienfeld investigated the spectra of the emission and showed that they were continuous. For a time OTR was referred to as Lilienfeld radiation. The theory of transition was developed by Ginzburg and Frank in 1945. They predicted that radiation would be produced by a charged particle traveling at constant velocity across the boundary between two media with different dielectric properties. The radiation was considered to be a special case of Cherenkov radiation. Goldsmith and Jelley observed TR from 1.5 MeV protons in 1958, but did not consider their results to be a definitive demonstration of the existance of transition ra-

diation. Gradually during the 1960 's, the experimental proof of the existance of OTR became established. Aitken et. al. observed and imaged the angular distribution of the OTR produced by $26-29 \mathrm{MeV}$ electron beams produced from the windows of a gas filled Cherenkov detector when the gas pressure was reduced to below the Cherenkov threshold condition. They also observed the polarization of the radiation, and raised the possibility of using the angular distribution to measure beam energy.

In the 1970's, Wartski intensively studied the properties of OTR and its application to charge particle beam diagnostics. He demonstrated that the angular distribution of OTR from a single foil could be used to measure the energy of of linac beams $(35-72 \mathrm{MeV})$, and that OTR images of the beam could be used to make high resolution beam profile measurements. He further showed how the interference pattern from a two foils OTR inteferometer could be used as a sensitive (1\%) beam energy diagnostic. Wartski experimentally investigated the effect of mutiple scattering on single foil and two foil interferometer radiation patterns.

In 1983, Fiorito and Rule of Naval Surface Warfare Center started to apply the techniques developed by Wartski to diagnosis high current electron beam. They studied the effect of beam divergence on the angular distribution pattern and polarization of OTR from a single foil and two foil interferometer. They applied the OTR emittance measurement techniques for electron beams from several linacs including both the Boeing and LANL HIBAF FEL accelerators. The result obtained from this technique is consistant with those from variable quadrupole method and mutiple screen method. 


\section{Transition radiation from single particle at normal incidence}

If a single particle of charge e crosses an interface (from the medium to the vacuum) at normal incidence, the intensity of the transition radiation emitted into the vacuum (forward emission), in a frequency range $d \omega$ and a solid angle $d \Omega$, is given by the following formula [3]:

$$
\frac{d^{2} W}{d \omega d \Omega}=\frac{e^{2} \beta^{2}}{\pi^{2} c} \frac{\sin ^{2} \theta \cos ^{2} \theta}{\left(1-\beta^{2} \cos ^{2} \theta\right)^{2}}\left|\frac{(\epsilon-1)\left[1-\beta^{2}-\beta\left(\epsilon-\sin ^{2} \theta\right)^{\frac{1}{2}}\right]}{\left[\epsilon \cos \theta+\left(\epsilon-\sin ^{2} \theta\right)^{\frac{1}{2}}\right]\left[1-\beta\left(\epsilon-\sin ^{2} \theta\right)^{\frac{1}{2}}\right]}\right|^{2}
$$

where $\epsilon$ is the complex dielectric constant of the medium, $\theta$ the angle of emission with respect to the direction of the charge velocity $\mathrm{v}$, and $\beta$ the particle velocity expressed in unit of the speed of light.

For extreme relativistic particles, and in the case where $|\epsilon| \gg 1$ as for a metal, the third term in (1) tends to unity and (1) reduces to

$$
\frac{d^{2} W}{d \omega d \Omega}=\frac{e^{2} \beta^{2}}{\pi^{2} c} \frac{\sin ^{2} \theta}{\left(1-\beta^{2} \cos ^{2} \theta\right)^{2}}
$$

The maximum intensity for (2) occurs at the angle,

$$
\begin{array}{r}
\sin \theta_{m}=\sqrt{\frac{1-\frac{1}{\gamma^{2}}}{\gamma^{2}}} \\
\theta_{m} \approx \gamma^{-1}
\end{array}
$$

where $\gamma$ is the Lorentz factor.

The OTR radiation is polarized in the plane of observation. The intensity of transition radiation depends strongly on the particle energy. It can be shown that the total intensity is proportional to $\gamma$ [5].

When the particle goes from vacuum to the medium, the intensity of the transition radiation emitted into the vacuum (backward emission) is easily obtained by changing $\beta$ to $-\beta$ in Eq. (1). For $\gamma \gg 1$, it takes the form,

$$
\frac{d^{2} W}{d \omega d \Omega}=\frac{e^{2} \beta^{2}}{\pi^{2} c} \frac{\sin ^{2} \theta}{\left(1-\beta^{2} \cos ^{2} \theta\right)^{2}}\left|\frac{\epsilon^{\frac{1}{2}}-1}{\epsilon^{\frac{1}{2}}+1}\right|^{2}
$$

which is just Eq. (1) times the Fresnel reflection formula for light normally incident on a medium. 


\section{The formation zone}

The formation zone of OTR radiation is defined as the distance measured along the particle tracjectory for which the phase difference between the radiation field and the Coulomb field of the particle is just equal to 1 radian,

$$
L_{m}=\frac{\beta c}{\omega}|1-\beta \sqrt{\epsilon} \cos \theta|^{-1}
$$

In a vacuum $\epsilon=1$ and

$$
L_{m}=\frac{\beta c}{\omega} \frac{1}{1-\beta \cos \theta}=\frac{\beta \lambda}{2 \pi} \frac{1}{1-\beta \cos \theta}
$$

for $\theta \ll 1$,

$$
L_{m} \approx \frac{\lambda}{\pi}\left(\gamma^{-2}+\theta^{2}\right)^{-1}
$$

for $50 \mathrm{MeV}$ electron, $L_{m}$ is about $0.16 \mathrm{~cm}$ at $\lambda=500 \mathrm{~nm}$.

\section{Transition radiation for particle at oblique incidence}

\subsection{Single particle theory}

The angular distribution for the transition radiation from a particle crossing the boundary from vacuum into medium at oblique incidence can easily be derived using image charge method [8].

As shown in figure 1, an electron is approaching the interface from left. The electron induces an positive image charge in the medium (the magnitude of its charge is not necessarily equal to that of electron). The image charge is moving in the direction of specular reflection. Both the electron and its image charge contribute to the radiation. The fourier transform of the magnetic field of the radiation is give by $[8]$,

$$
C(\omega)=\frac{\beta \times \hat{n}_{0}}{1-\beta \cdot \hat{n}_{0}}+r \frac{\beta \times \hat{n}}{1-\beta \cdot \hat{n}}
$$

where $r$ is the Fresnel reflection coefficient for the medium. $\hat{n}_{0}$ is along the direction of observation. $\hat{n}$ is the reflection direction corresponding to $\hat{n}_{0}$. The two vectors define the plane of observation. The first term corresponds to the 
radiation from the electron, and the second term is that from the image charge.

If the plane of incidence does not coincide with the plane of observation, the radiation is circularly polarized. Let $C_{\|}(\omega)$ be the magnetic field component perpendicular to the plane of observation and $C_{\perp}(\omega)$ the component parallel to the plane of observation. Assuming the two planes nearly coincide and the particle is relativistic and approximately $45^{\circ}$ incident, we have [8]:

$$
\begin{aligned}
& C_{\|}(\omega)=\beta_{\|} r_{\|} \frac{\sin \theta}{1-\beta_{\|} \cos \theta}+\frac{\beta_{\|} \cos \theta}{1-\beta_{\|} \sin \theta} \\
& C_{\perp}(\omega)=\beta_{\perp} r_{\perp} \frac{1}{1-\beta_{\|} \cos \theta}+\frac{\beta_{\perp}}{1-\beta_{\|} \sin \theta}
\end{aligned}
$$

where $\beta_{\|}\left(\beta_{\perp}\right)$ is the component of $\beta$ parellel (perpendicular) to the plane of observation, $\theta$ is the angle between the reflection of $\beta$ and $\hat{n}_{0}$ projected into the plane of observation (the angle between the reflection of $\beta_{\|}$and $\hat{n}$ ), $r_{\|}\left(r_{\perp}\right)$ is the Fresnel coefficient for the particular polarization. Using small angle approximation for sinusoidal function, it can be shown that the intensity per unit frequency, per steradian, is given by [8],

$$
\begin{aligned}
& \frac{d^{2} W_{\|}}{d \omega d \Omega} \approx \frac{e^{2}}{c} \frac{\beta_{\|}^{2}}{\pi^{2}}\left[\frac{\left|r_{\|}\right|^{2} \theta^{2}}{\left(\gamma^{-2}+\theta^{2}\right)^{2}}+\frac{\operatorname{Rer}_{\|} \theta}{\gamma^{-2}+\theta^{2}}\right] \\
& \frac{d^{2} W_{\perp}}{d \omega d \Omega} \approx \frac{e^{2}}{c} \frac{\beta_{\perp}^{2}}{\pi^{2}}\left[\frac{\left|r_{\perp}\right|^{2}}{\left(\gamma^{-2}+\theta^{2}\right)^{2}}+\frac{R e r_{\perp}}{\gamma^{-2}+\theta^{2}}\right]
\end{aligned}
$$

The intensity distribution for the parallel component around the reflection of $\beta_{\|}$exhibits a similar characteristic as the case with normal incident, which has a maximun intensity at $\theta_{m}=\frac{x}{\gamma}$. Nevertheless, the pattern of OTR is no longer symmetric because $r_{\|}\left(\left|r_{\|}\right|^{2}\right)$ is not symmetric as $\theta$ changes sign.

In figure 2, we plot the intensity distribution for particles with different energy and $45^{\circ}$ incident observed in the plane of incidence at $\lambda=500 \mathrm{~nm}$.

\subsection{Theory for an ensemble of particles}

We will assume a beam of relativistic particles with its transverse divergence distribution given by,

$$
P\left(\alpha_{x}, \alpha_{y}\right)=\left(2 \pi \sigma_{x}^{2}\right)^{-\frac{1}{2}} e^{-\frac{a_{x}^{2}}{2 \sigma_{x}^{2}}} \cdot\left(2 \pi \sigma_{y}^{2}\right)^{-\frac{1}{2}} e^{-\frac{\alpha_{y}^{2}}{2 \sigma_{y}^{2}}}
$$


incidents on the interface in such a way that the centroid particle has a $45^{\circ}$ incident angle and the $\mathrm{x}$-direction ( $\mathrm{y}$-direction) is parallel (perpendicular) to the plane of incidence of the centroid particle. Both $\mathrm{x}$-direction and $\mathrm{y}$-direction are perpendicular to the path of the particle beam. $\sigma_{x}$ and $\sigma_{y}$ in Eq.(14) are rms angle of beam divergence in the $\mathrm{x}$ and $\mathrm{y}$ directions. Furthermore, we take the plane of incidence of the centroid particle as our plane of observation.

The effect of beam divergence on the transition radiation pattern can be found by averaging over the Gaussian distribution, and is give by [7],

$$
\begin{aligned}
& \frac{d^{2} \bar{W}_{\|}}{d \omega d \Omega}=\frac{e^{2}}{c} \beta^{2}(2 \pi)^{-\frac{3}{2}} \sigma_{x}^{-1}\left\{| r _ { \| } | ^ { 2 } \operatorname { R e } \left[\left(\gamma+\frac{\sqrt{2} Z}{\sigma_{x}}\right)\right.\right. \\
&\left.\left.\times W(Z)-\sigma_{x}^{-1}\left(\frac{2}{\pi}\right)^{\frac{1}{2}}\right]-2 \operatorname{Re}\left(r_{\|}\right) \operatorname{Im} W(Z)\right\} \\
& \frac{d^{2} \bar{W}_{\perp}}{d \omega d \Omega}=\frac{e^{2}}{c} \beta^{2}(2 \pi)^{-\frac{3}{2}} \sigma_{x}^{-1} \sigma_{y}^{2} \gamma^{2}\left|r_{\perp}\right|^{2} \\
& \times R e\left[\left(\gamma-\frac{\sqrt{2} Z}{\sigma_{x}}\right) W(Z)+\sigma_{x}^{-1}\left(\frac{2}{\pi}\right)^{\frac{1}{2}}\right]
\end{aligned}
$$

where the complex variable $Z \equiv\left(\gamma^{-1}+i \theta\right) /\left(\sqrt{2} \sigma_{x}\right)$ and $W(Z)$ is diefined in terms of the complex error function $\Phi(Z)$ as $W(Z)=[1-\Phi(Z)] \exp \left(Z^{2}\right)$. In eq.(16), we have assumed that $\sigma_{x}, \sigma_{y} \ll 1 / \gamma$, and have neglected the contribution from the second term in eq.(12).

Eq.(15) and (16) suggest a way to measure the beam emittance at a single location. One can focus the particle beam such that the beam waists in both the $x$ and $y$ direction occur at the interface, then measure the intensity distributions for the two direction of polarization $(1, \|)$ in the plane of incidence of the centroid particle. The patterns can be analyzed by fitting them using eq.(15) and (16) with $\sigma_{x}$ and $\sigma_{y}$ as the fitting parameters. The rms dimension $x_{r m s}$ and $y_{r m s}$ will also be measured by adjusting the focus of the lens. The rms emittance in each corresponding direction can then be obtained by mutiplying the rms divergence by the rms dimension.

$$
\begin{aligned}
& \epsilon_{r m s, x}=\sigma_{x} x_{r m s} \\
& \epsilon_{r m s, y}=\sigma_{y} y_{r m s}
\end{aligned}
$$

Distribution patterns for difference beam divergence are ploted in figure 3 . It can be seen, as the beam divergence increases, radiation starts to leak into the $1 / \gamma-$ cone and eventually smear out the pattern. 


\section{Two-foil transition radiation interferome- ter}

\subsection{Single particle theory}

The two-foil transition radiation interferometer (OTRI), which invented by Wartski [3], consists of two parallel foils placed at an angle of approximately $45^{\circ}$ with respect to an electron beam. Particles exiting the back of the first foil produce transition radiation in the optical region which is reflected by the front surface of the second foil, as shown in figure 4 . The second foil also produces transition radiation which is coherent with respect to the transition radiation from the first foil, and thus an interference pattern is produced which is centered around the direction of specular reflection. The phase difference between the transition on the first and second foils is given by,

$$
\phi=\left(\frac{2 \pi L}{\lambda \beta}\right)(1-\beta \cos \theta)=\frac{L}{L_{m}}
$$

where $L$ is the seperation between the foils in the path where the electron travels, $L_{m}$ is the vacuum formation length and $\theta$ is the same as defined previously.

The amplitude of the magnetic field of the radiation can be written as $[8]$,

$$
C(\omega)=r \frac{\beta \times \hat{n}}{1-\beta \cdot \hat{n}}-e^{-i \phi} \cdot r \frac{\beta \times \hat{n}}{1-\beta \cdot \hat{n}}
$$

The first term in the above equation corresponds to the contribution from the image charge in the second foil. The second term corresponds to the radiation from the back of the first foil after it is reflected from the second surface. we have neglected the radiation from the real charge at the second surface. With assumption similar to that of single foil calculation, the intensity distribution in the plane of observation is [8],

$$
\begin{aligned}
& \frac{d^{2} W_{\|}}{d \omega d \Omega}=\frac{e^{2}}{c} \frac{\beta_{\|}^{2}}{\pi^{2}} \frac{\left|r_{\|}\right|^{2} \theta^{2}}{\left(\gamma^{-2}+\theta^{2}\right)^{2}} \cdot \sin ^{2}\left[\frac{\pi L}{2 \lambda}\left(\gamma^{-2}+\theta^{2}\right)\right] \\
& \frac{d^{2} W_{\perp}}{d \omega d \Omega}=\frac{e^{2}}{c} \frac{\beta_{\perp}^{2}}{\pi^{2}} \frac{\left|r_{\perp}\right|^{2}}{\left(\gamma^{-2}+\theta^{2}\right)^{2}} \cdot \sin ^{2}\left[\frac{\pi L}{2 \lambda}\left(\gamma^{-2}+\theta^{2}\right)\right]
\end{aligned}
$$

The interference order of the fringes is given by,

$$
p=\frac{\phi}{2 \pi}=\frac{L}{2 \pi L_{m}}
$$


for relativistic particle,

$$
p=\left(\frac{L}{2 \lambda}\left(\gamma^{-2}+\theta^{2}\right)\right.
$$

The value if $p$ is a minimun at the center of the pattern $(\theta=0)$

$$
p_{0} \approx\left(\frac{L}{2 \lambda}\right) \gamma^{-2}
$$

the value of $p_{0}$ is generally fractional and thus the center of the pattern corresponds th neither a maximum nor minimum. The intensity maxima occur at $p=k+1 / 2$ and minima at $p=k$, where $\mathrm{k}$ is an integer. The angles for intensity maxima and minima occur at

$$
\theta=\left[\left(\frac{2 \lambda}{L}\right)\left(p-p_{0}\right)\right]^{\frac{1}{2}}, p=k+0.5, o r, p=k
$$

thus the interference pattern can be used to determine $p_{0}$ and hence $\gamma$. Wartski have used the interference pattern to determine $\gamma$ to $1 \%$ accuracy.

\subsection{Theory for an ensemble of particles}

The effect of beam divergence on the inteference pattern of OTRI can be analyzed using treatment similar to the single foils case. The resulting angular intensity distribution in the plane of incidence for the centroid particle is given by,

$$
\begin{aligned}
& \frac{d^{2} \bar{W} W_{\|}}{d \omega d \Omega}=\int_{-\infty}^{+\infty} \int_{-\infty}^{+\infty} \frac{d^{2} W_{\|}}{d \omega d \Omega}\left(\theta-\alpha_{x}, \alpha_{y}\right) P\left(\alpha_{x}, \alpha_{y}\right) d \alpha_{x} d \alpha_{y} \\
& \frac{d^{2} \bar{W}_{\perp}}{d \omega d \Omega}=\int_{-\infty}^{+\infty} \int_{-\infty}^{+\infty} \frac{d^{2} W_{\perp}}{d \omega d \Omega}\left(\theta-\alpha_{x}, \alpha_{y}\right) P\left(\alpha_{x}, \alpha_{y}\right) d \alpha_{x} d \alpha_{y}
\end{aligned}
$$

The consequence of the beam divergence is the smearing of the interference pattern.

So divergence can be measured by focusing the beam to a waist at the second foil. There are two ways to determine the divergence of the particle beam.

The first method is to record the angular intensity pattern resulted from the interference of a particular wavelength in the plane of incidnece of the centroid particle for the two direction of polarization mentioned. The recorded data can then be fitted to patterns resulted from Gaussian distribution in divergence in both direction with the rms divergence as a fitting 
parameters. Once the rms divergence is determined, one can then calculated the rms emittance by mutiplying the rms divergence with the rms beam size.

In figure 5 , we plot the distribution from transition radiation interferometer for $50 \mathrm{MeV}$ beam with two different divergence.

The second method is to measure the interference intensity as a function of wavelength at a particular angle. The effect of divergence on the wavelength interference pattern is similar to the angular interference pattern. Finite divergence of the beam will smear out the pattern. The same fitting procedure as discussed in the first method can be applied to determine the divergence of the beam.

The sensitivity of the two method will be discuss later in this report.

\section{Using transition radiation to charactize the electron beam}

\subsection{Intensity of transition radiation}

The rate of transition radiation is about 1 visible photon for every 500 particles crossing the boundary. For a $1 \mathrm{nC}$ electron bunch, about $10^{7}$ visible photons are generated.

According to OTR experiments performed at the LANL HIBAF FEL facility ( $\sim 20 \mathrm{MeV}$ linac) and the Boeing visible FEL facility ( $\sim 110 \mathrm{MeV}$ linac) [2], a single e-beam micropulse of about 3 to $5 \mathrm{nC}$ charge and $15-17 \mathrm{MeV}$ in energy can be imaged via OTR by an intensified TV camera [ either silicon intensified traget (SIT) or gated, intensified charge-injection-device (ICID) ]. Standard vidicons was able to observe OTR beam spots at $100 \mathrm{Mev}$, at the Boeing FEL, but it was necessary to integrate the OTR From about 150 micropulses ( $1.5 \mathrm{nC} /$ micropulse) to obtain good beam images. Experiments which incorporated two cameras and beam splitter to allow simultaneously monitoring of the beam image and angular distribution pattern at a single lacation was also performed.

In order to observe OTR from a single micropulse from the ATF linac, which has about $1 \mathrm{nC}$ charge, a intensified CCD camera is necessary. However, if multi-pulse trains can be produced (for example, the 100-pulse system for the visible FEL), a CCD camera without intensifier can produce good image of the OTR by integrating over the pulse train. 


\subsection{Spatial resolution of transition radiation BPM}

Altough the issues concerning the size of transition radiation source and formation remain controversial, clearer particle beam images had been produced from OTR than that produced from phospher screens. Wartski observed sharper beam images in TR than from fluorescent zinc sulfide screens [4]. In both Boeing and LANL, by replacing phosphor screens previously used in the wiggler, the resolution of the beam images was improved by approximately a factor of five, and the beam could be better matched to the size required by wiggler transport characteristics [2].

According to Rule and Fiorito [11], transition radiation should occur exactly at the point where the electron crosses the interface. This means the spatial resolution limit of OTR BPM will only be limitted by the optical system. The main contributions in spatial resolution from optical system are the size of the pixels of the CCD camera and the use of intensifier. The use of image intensifier can worsen the resolution by a factor of two comparing to the case without an image intensifier.

\subsection{Using OTR for electron energy measurement}

As discussed earlier, the angular intensity distribution of transition radiation depends strongly on the energy of the radiating particle. This information can be analyzed to obtain the energy of the particle.

The angular distribution can be detected by having the focus point of the camera lense concide with the point where the particle hits the interface.

For transition radiation from a particle crossing a single interface normally, both the forward and backword radiation exhibit azimuthal symmetry and the intensity peaks at angle $\theta \approx 1 / \gamma$ from the normal direction.

For particle acrossing the interface at $45^{\circ}$, the backward radiation will be around the direction of specular direction, which is normal to the path of the particle. This arrangement makes it very convenient to detect the radiation and also minimizes unwanted backgroud radiation, especially bremsstrahlung, which is concentrated around the forward direction.

In the plane of incidence, the radiation is polarized parallel to the plane of incidence and peaks at angle $\theta_{m} \approx 1 / \gamma$ from the direction of specular reflection.

By mesuring the angle at whcih the maximun intensty occurs, one can also known the energy of the particle. The accuracy of the method is limitted by two factors. The first is due to resolution of the detection system. The 
second is due to the fact that the effects of energy and finite divergence of a particle beam are convoluted. The rms divergence of the beam limits the smallest angular resolution. The relative accuracy of the energy measurement due to beam divergence only is then give by,

$$
\frac{\delta \gamma}{\gamma}=\frac{\sigma_{\|}}{\theta_{m}}
$$

where $\sigma_{\|}$is rms divergence in the direction parallel to the incident plane of the centroid particle and $\theta_{m}$ is the angle where maximun intensity occurs. In order to minimize this contribution to the error in energy meaurement, it is critical to minimize the divergence of the beam.

Compared with single foil transition radiation, two foil tansition radiation interferometer are more sensitive for energy measurement. As discussed before, the angle of the interference maxima or minima for a particular wavelength of transition radiation is determined by the energy of the particle. Although the two foil method sufers the same limitations in angle measurement, the redundant amount of data provides a better determination of energy. Wartski had achieved a presicion of about $1 \%$ in measuring $\gamma$ using this method $[3]$.

\subsection{The resolution of electron beam divergence mea- surement}

The resolution for beam divergence measurement is determined by many factors, for example, the resolution of the detection system and the energy spread of the beam.

For angle $\theta \ll \theta_{m}$, the relative intensity varies as,

$$
\frac{I(\theta)}{I\left(\theta_{m}\right)} \approx \frac{(\theta \gamma)^{2}}{4}
$$

Assume that our detection system has an intensity resolution of $\eta$, then the resoltion in divergence due to detection system is,

$$
\theta_{\min } \approx \sqrt{4 \eta} \frac{1}{\gamma}
$$

For a 8 -bit detection system,

$$
\theta_{\min } \approx 12.5 \% \frac{1}{\gamma}
$$


The analysis appears to be consistant with the experiment results for single foil transition by Fiorito and Rule. They conclude that beam divergence of the order of $15 \%$ of $\theta_{m} \sim 1 / \gamma$ could be measured.

Similar analysis can be performed for two-foil interferometer. We assume the divergence of the beam is a lot smaller than $1 / \gamma$ and the observation angle is about $1 / \gamma$. We have

$$
\theta_{\min } \approx \sqrt{\eta} \frac{\gamma \lambda}{\pi L}
$$

If we express the interfoil distance in unit of the formation zone length along the path of the centroid particle,

$$
L=k \frac{\lambda \gamma^{2}}{\pi}
$$

where $k$ is a real number. Then,

$$
\theta_{\min } \approx \frac{\sqrt{\eta}}{k} \frac{1}{\gamma}
$$

In principle, arbitrarily low divergence can be measure by increased the interfoil distance. However, as $\mathrm{L}$ increases, the spacing between interference maxima and minima will decrease and the resolution of the measurement will be limitted by angular resolution.

Caution has to be taken when chosing the first foil. The thickness has to be chosen in such a way that the change in divergence due to mutiple scattering while the beam passing through the foil is a lot smaller than the intrinsic divergence of the beam. The formula for calculating the angle change from mutiple scattering is given in appendix.

The resolution in divergence for transition radiation spectrometer should be the same as transition radiation spectrometer for the same inter-foil spacing because the geometry is the same. However, one can use a longer L for transition radiation spectrometer. It turns out that even for relative long $\mathrm{L}$, the spacing between interference peak in the optical light spectrum is still a lot larger than the resolution of the optical spectrometer. So one can conclude that transition radiation spectrometer could achieve a finer resolution in divergence measurement than transition radiation interferometer.

\section{Possible OTR experiments at ATF}

The Accelerator Test Facility (ATF) at BNL is a users' facility for experiments in accelerator and beam physics. It is equipped with a high bright- 
ness photocathode rf-gun, two SLAC linac sections, high-power picosecond pulse lasers synchronized with the electron beam and a variety of diagnostic equipment. Recently, a solenoid is installed at the low energy end to compensate the emittance growth due to space charge. According to simulation [14], with proper adjustment of solenoid field, the phase and amplitude of the gun and the linac, the laser spot size of the photocathode, very low emittance electron beam can be obtain. The unique characteristic of the ATF electron beam provides an ideal test-bed for the development of transition radiation based diagnostics. We plan to carry out a complete experimental program at ATF to develop transition radiation based diagnostics.

\subsection{Low energy end}

An aluminum mirror has been placed at the location after the exit of the electron gun. The mirror can be push in or pull out of the path of the electron beam by a manual actuater. When the mirror is pushed in, the electron beam will hit the mirror with approximate $45^{\circ}$ incident. Transiton radiation will be produced nearly normal to the electron beam coming out of an optical window. The main purpose of the setup is to measure the electron beam energy and study the characteristic of transition in order to gain experience for further applying transition radiation devices to electron beam diagnostic at the high energy beamlines.

We will study the angular intensity distribution and deduce the energy of the electron coming out of the gun. Because of the relative low energy of the electron $(\gamma \approx 9)$, transition radiation will spread over a large angle. It will be difficult to design an optical system to cover the whole angular distribution with a single camera, so we decide to use a photomutiplier combined with a pinhole as a detecter. The photomutiplier will be mounted on a translational stage. The distribution will be mapped out by changing the location of the photomutiplier.

We will also try to image the electron beam profile using transiton radiation. The electron beam image can be obtained by abjusting the focus of the camera lens. Because the relative low energy of the electron, the intensity of the transition will be weak and disperse in wide range. Only a small portion of radiation can be collected for beam imaging. This makes it necessary to use an image intensifier. 


\subsection{Visible FEL beamline}

In conventional emittance measurement method using BPMs, for example, variable quadrupole method, two or three-screen method $[20][21][22]$, the accuracy of the measurement is critically related to resolution of the BPMs. In the visible FEL experiment beamline of ATF, prior to wiggler installation, the three-screen method will be used to characterize the electron beam. One of the BPM will be placed at the location where the center of the wiggler is supposed to be and the other two BPMs will be placed about 1 meter upstream and downstream of the center BPM. We calculated the accuracy of the emittance measurement for a $50 \mathrm{MeV}$ electron beam with an normalized rms emittance of $5 \mathrm{~mm} \cdot \mathrm{mrad}$, and the waist of the beam coincides with the center BPM, as a function of the size of the beam waist for different resolution of the BPM. The result is shown in figure 6 .

In order to obtain accurate result, it is important to have high resolution BPMs. Phosphor screen has a resolution of about 50-100 $\mu \mathrm{m}$. Figure 6 . shows us that the resoltion of phsphor screens is not good enough to achieve the goal. To reduce the error, it is necessary to use transition radiation BPM screens, which could have better resolution as discussed previously.

We plan to replace the two BPMs at up and down stream with aluminum coated pellicle and the center BPM with a aluminum mirror or aluminum foil which is mounted on an actuator. These should be sufficient enough for us to characterize the beam.

We also plan to carry out transition radiation two-foil interferometer and two-foil spectrometer study at the center location. FEL beamline provides an ideal environment for the experiment. First of all, a He-Ne laser exists at the beamline for FEL optical allignment, and can be used for alignment of the transition radiation screens. Second, an optical transport line, which is designed for optical wavelength, is already in place to transport FEL light from this beamline to an optical spectrometer in the FEL room. This again can be readily made us in our two-foil transtion radiation spectrometer study.

\subsection{HGHG FEL beamline}

Similarly three-screen method will also be used to characterize the electron at the HGHG FEL beamline. We can also do variable quadrupole emittance measurement utilizing transition BPMs.

Detail transition radiation experiment program at beamline 2 will be determined as time evolves. 


\subsection{Other opportunities at ATF}

ATF has extensive experimental programs in high gradient laser acceleration. In order to carry out the experiment successfully, an energy spectrometer with large acceptance and high resolution will be needed to analyze the accelerated electrons. It is difficult and expensive to design a conventional magnetic dipole spectrometer which would satisfy both requirement. Because of the sensitivity of transition radiation responding to the energy of the radiating particle, we are motivated to consider the possiblity of using transition radiation to analyze particle energy spectrum. Intensive study is underway.

\section{Acknowledgements}

The authors wish to thank Dr. R. Fernow for providing helpful references in understanding the physics of transition radaition, Dr. K. Batchelor and Dr. T. Rao for useful discussions.

\section{Appendix}

\section{A Three-screen measurement method}

The following discussion of error propagation in three-screen emittance measurement is based on [22] To meausure the beam phase space, one can meause the beam size using three profile monitors, equally spaced by the distance $L$, as shown in figure a. Defining the origin to be at the center profile montor, so that $z_{1}=-L, z_{2}=0$,and $z_{3}=+L$, and the beam waist occurs at $z_{0}$ with beam size $x_{0}$ and divergence $\theta_{0}$. Then,

$$
\begin{array}{r}
x_{1}^{2}=x_{0}^{2}+\left(L+z_{0}\right)^{2} \theta_{0}^{2} \\
x_{2}^{2}=x_{0}^{2}+z_{0}^{2} \theta_{0}^{2} \\
x_{3}^{2}=x_{0}^{2}+\left(L-z_{0}\right)^{2} \theta_{0}^{2}
\end{array}
$$

Thus, knowing the beam size on the profiles monitors and the profile monitor seperation $L$, we can calculate the waist size, the divergence at the waist, and the location of the waist.

$$
x_{0}=\left\{x_{2}^{2}-\frac{1}{8}\left(\frac{x_{1}^{2}-x_{3}^{2}}{x_{1}^{2}-2 x_{2}^{2}+x_{3}^{2}}\right)\right\}^{\frac{1}{2}}
$$




$$
\begin{array}{r}
\left.\theta_{0}=\frac{1}{\sqrt{2} L}\left(x_{1}^{2}-2 x_{2}^{2}+x_{3}^{2}\right)\right)^{\frac{1}{2}} \\
z_{0}=\frac{L}{2}\left(\frac{x_{1}^{2}-x_{3}^{2}}{x_{1}^{2}-2 x_{2}^{2}+x_{3}^{2}}\right)
\end{array}
$$

The beam emittance $\epsilon$ is given by $x_{0} \theta_{0}$,

$$
\epsilon=\frac{1}{4 L}\left[8 x_{2}^{2}\left(x_{1}^{2}-2 x_{2}^{2}+x_{3}^{2}\right)-\left(x_{1}^{2}-x_{3}^{2}\right)^{2}\right]^{\frac{1}{2}}
$$

To quantitatively determine the uncertainty in the phase space measurements, standard propagation of error calculation have been made. In make these calculation, it has been assumed that the uncertainty in the measured beam size $\sigma_{x i}$ dominate, and that $\mathrm{L}$ is know to greater precision. The results are,

$$
\begin{array}{r}
\frac{\sigma_{x_{0}}}{x_{0}}=\left\{\sigma_{x_{1}}^{2} x_{1}^{2}\left(x_{1}^{2}-x_{3}^{2}\right)^{2}\left(x_{1}^{2}-4 x_{2}^{2}+3 x_{3}^{2}\right)^{2}\right. \\
+4 \sigma_{x-2}^{2} x_{2}^{2}\left[4\left(x_{1}^{2}-2 x_{2}^{2}+x_{3}^{2}\right)^{2}-\left(x_{1}^{2}-x_{3}^{2}\right)^{2}\right]^{2} \\
\left.+\sigma_{x-3}^{2} x_{3}^{2}\left(x_{1}^{2}-x_{3}^{2}\right)^{2}\left(3 x_{1}^{2}-4 x_{2}^{2}+x_{3}^{2}\right)^{2}\right\}^{\frac{1}{2}} \\
\quad / 4 \sqrt{2} x_{0}^{2}\left(x_{1}^{2}-2 x_{2}^{2}+x_{3}^{2}\right)^{2} \\
\frac{\sigma_{t h e t a_{0}}}{\theta_{0}}=\sqrt{2} \frac{\left(\sigma_{x_{1}}^{2} x_{1}^{2}+4 \sigma_{x_{2}}^{2} x_{2}^{2}+\sigma_{x_{3}}^{2} x_{3}^{2}\right)^{\frac{1}{2}}}{\left(x_{1}^{2}-2 x_{2}^{2}+x_{3}^{2}\right)} \\
\sigma_{z_{0}}=\frac{2 L}{\left(x_{1}^{2}-2 x_{2}^{2}+x_{3}^{2}\right)}\left[\sigma_{x_{1}}^{2} x_{1}^{2}\left(x_{2}^{2}-x_{3}^{2}\right)^{2}\right. \\
\left.+\sigma_{x_{2}}^{2} x_{2}^{2}\left(x_{1}^{2}-x_{3}^{2}\right)^{2}+\sigma_{x_{3}}^{2} x_{3}^{2}\left(x_{1}^{2}-x_{2}^{2}\right)^{2}\right]^{\frac{1}{2}}
\end{array}
$$

and

$$
\begin{array}{r}
\frac{\sigma_{\epsilon}}{\epsilon}=\frac{4}{\sqrt{2}\left[8 x_{2}^{2}\left(x_{1}^{2}-2 x_{2}^{2}+x_{3}^{2}\right)-\left(x_{1}^{2}-x_{3}^{2}\right)^{2}\right]} \\
\times\left[\sigma_{x_{1}}^{2} x_{1}^{2}\left(x_{1}^{2}-4 x_{2}^{2}-x_{3}^{2}\right)^{2}+16 \sigma_{x_{2}}^{2} x_{2}^{2}\left(x_{1}^{2}-4 x_{2}^{2}+x_{3}^{2}\right)^{2}\right. \\
\left.+\sigma_{x_{3}}^{2} x_{3}^{2}\left(x_{1}^{2}+4 x_{2}^{2}-x_{3}^{2}\right)^{2}\right]^{\frac{1}{2}}
\end{array}
$$

It is always best to have the waist near the center monitor in order to obtain minimun emittance measure error. 


\section{B Mutiple scattering for en electron travers- ing in matter}

The value of the space angle due to mutiple scattering of the electron at $1 / \mathrm{e}$ if its peak value is given by the following formula:

$$
\theta_{e}=\frac{17.5[\mathrm{rad}]}{\beta p[M e v / c]} \sqrt{\frac{L}{L_{R}}}\left[1+0.125 \log _{10}\left(\frac{10 L}{L_{R}}\right)\right]
$$

where $L_{R}$ is the radiation length of the matter and $L$ is the thickness. For aluminum, $L_{R}=8.9 \mathrm{~cm}$.

For $L=1 \mu m$ and $50 \mathrm{Mev}$ electron,

$$
\theta_{e} \approx \frac{59.4}{\gamma}[\operatorname{mrad}]
$$

For $L=25 \mu m$

$$
\theta_{e} \approx \frac{0.4}{\gamma}[r a d]
$$

\section{Error propagation in angle determination}

In OTR experiment, accurate determation of angle is crucial for characterization of beam parameter. With configuration as shown in figure a,

$$
\begin{array}{r}
\tan \theta=\frac{b}{a} \\
\theta=\arctan \left(\frac{b}{a}\right)
\end{array}
$$

The error in determining $\theta$ can be calculate from the standard theory of error propagation.

$$
\sigma_{\vartheta}=\frac{b}{a} \sqrt{\left(\frac{\sigma_{a}}{a}\right)^{2}+\left(\frac{\sigma_{b}}{b}\right)^{2}}
$$

\section{References}

[1] V. L. Ginzburg, Radiation By Uniformly Moving Sources, The Lesson Of Quantum Theory, J. de Boer, E. Dal And O. Ulfbeck, ed., Elsevier Science Publisher B. V. (1986) 
[2] Ralph B. Fiorito and Donald W. Rule, AIP Conference Proceeding No. 319: Accelerator Instrumentation,Fifth Annual Workshop, October 2023,1993, Sante Fe, NM, R. Shafter, ed.

[3] L. Wartski, et. al., Interference Phenomenon In Optical Transition Radiation And Its Application To Particle Beam Diagnostic And Mutiple Scattering Experiments, J. Appl. Phys., V. 46 ,3644 (1975)

[4] L. Wartski, et. al., Detection Of Optical Transition Radiation And Its Application To Beam Diagnostic, IEEE Transactions In Nuclear Science, V. 20,544 (1973)

[5] D. W. Rule and R. B. Fiorito, The Use Of Transition Radiation As A Diagnostic For Intense Beams, Naval Surface Warfare Center Technical Report, NSWC TR 84-134 (1984)

[6] B. Dolgoshein, Transition Radiation Detector', Nucl. Inst. And Meth. A326,434 (1993)

[7] D. W. Rule, Transition Radiation Diagnostic For Intense Charged Particle Beams, Nucl. Inst. And Meth. B24/25, 901 (1987)

[8] D. W. Rule, et. al., Comparative Analysis Of Optical Transition Radiation-Based Electron-Beam Emittance Measurements For The Los Alamos Free Electron Laser, Nucl. Instr. And Meth. A296, 739 (1990)

[9] M. L. Ter-Mikaelian, High-Energy Electromagnetic Processes In Condensed Media, Wiley-Interscience,New York (1972)

[10] Alex H. Lumpkin, Advanced, Time-resolved Imaging Technoques For Electron-beam Characterizations, AIP Conf. Proc. No. 229, 151, E.S. McCrory,ed.,N.Y.,1991

[11] D. W. Rule and R. B. Fiorito, Imaging Micron Sized Beams With Optical Transition Radiation, Proc. Of The 1993 Particle Accelerator Conference, Washington,DC, 17-20, May,1993

[12] Ilan Ben-Zvi, The BNL Accelerator Test Facility And Experimental Program, BNL-47785

[13] J. C. Gallardo and H. G. Kirk, An Injection Scheme For The Brookhaven ATF Utilizing Space-charge Emittance Growth Compensation, Proceeding 
Of The 1993 Particle Accelerator Conference, Washington, DC, 36153617, May, 1993

[14] J. C. Gallardo and H. G. Kirk, Optimization Of The Brookhaven ATF Inline-injection System Utilizing Parmela, Unpublished

[15] A. van Steenbergen, electron Beam Optics For The FEL Experiment And IFEL Experiment, BNL-46840

[16] X.J. Wang and H. G. Kirk, The Brookhaven ATF Low-emittance Beam Line, BNL-46190

[17] X. Z. Qiu, Electron Beam Transport Study For The High Gain Harmonic Generation Free Electron Laser At ATF, Unpublished

[18] A. van Steenbergen, Private Communication

[19] R. Fernow, Introduction To Experimental Particle Physics, Cambridge University Press, Cambridge, England (1986)

[20] X.J. Wang, et. al., Automatic Emittance Measurement At The ATF, Proc. Of The 1993 Particle Accelerator Conference, Washington, DC, 17-20,May, 1993

[21] K. T. McDonald and D. P. Russell, In Proc. Of The Joint US-CERN School On Observation, Diagnostic, And Correction In Particle Beams, Capri(1988)

[22] K. D. Jacobs, et. al., Emittance Measurements At The Bates Linac, Presented At The 1989 Particle Accelerator Conference, Chicago, I.L.,20-23 March, 1989

[23] A. C. Melissinos, Experiments In Modern Physics, Academic Press,San Diego (1966) 


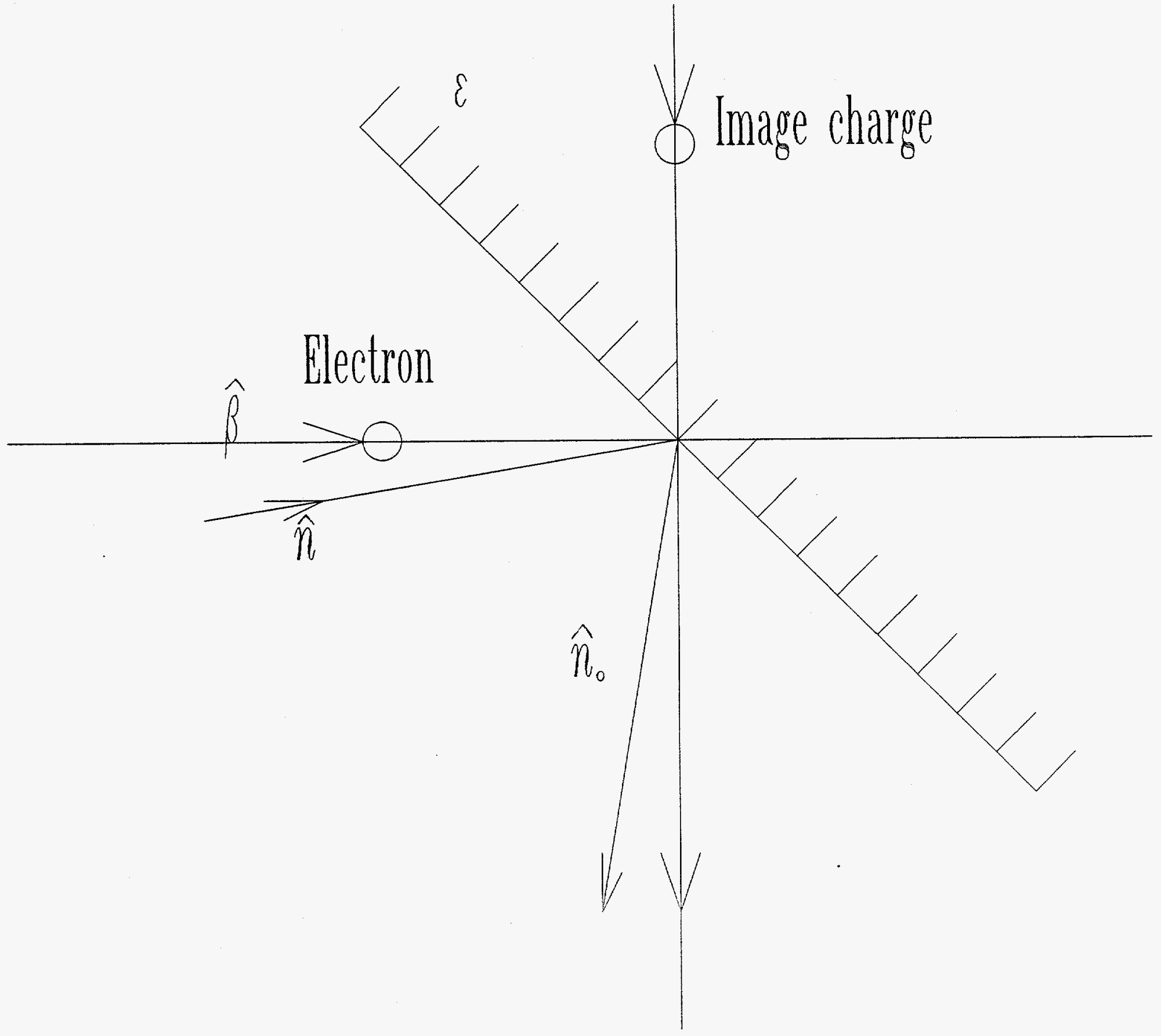

Figure 1. Transition radiation from single boundary 


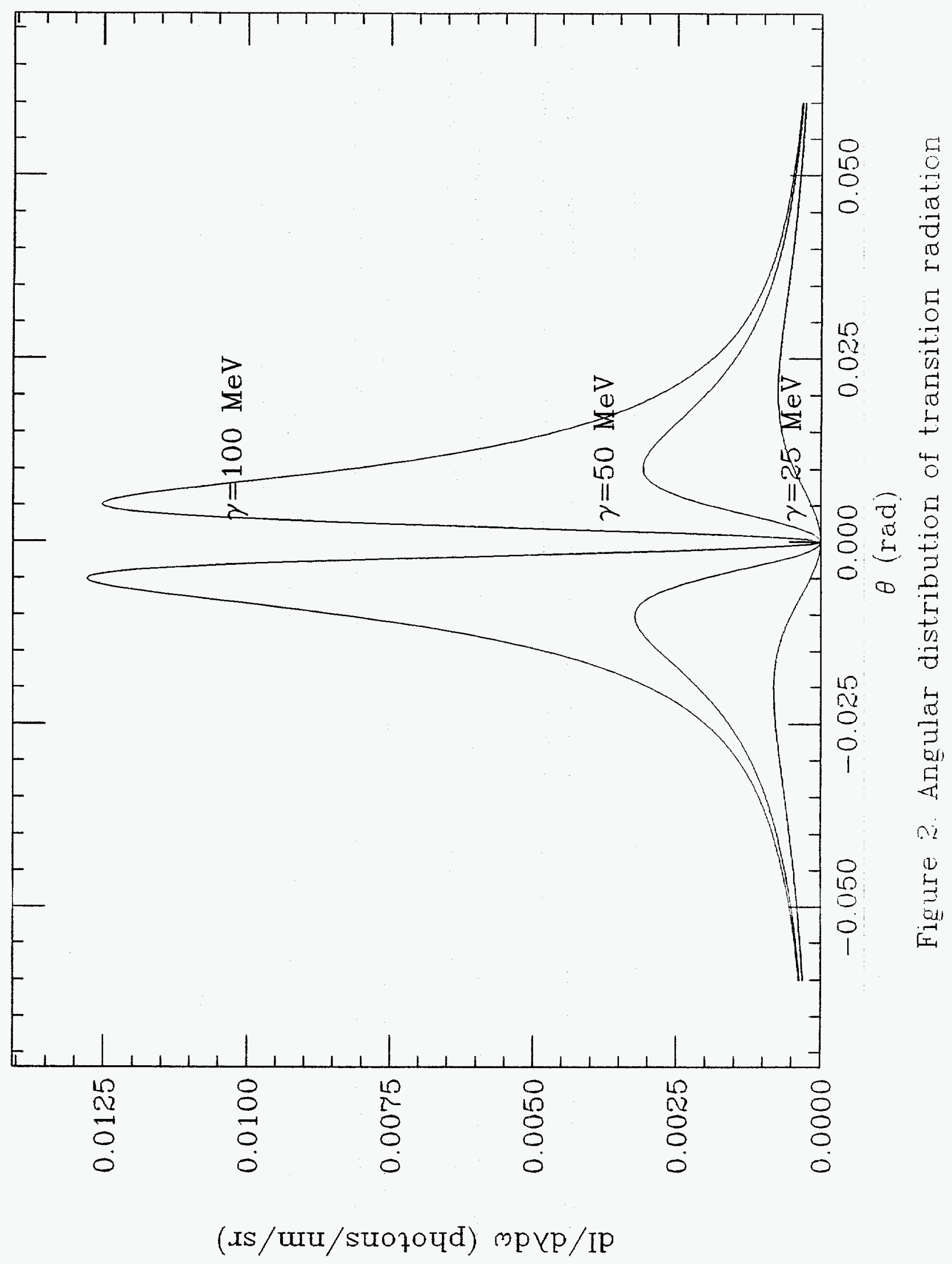




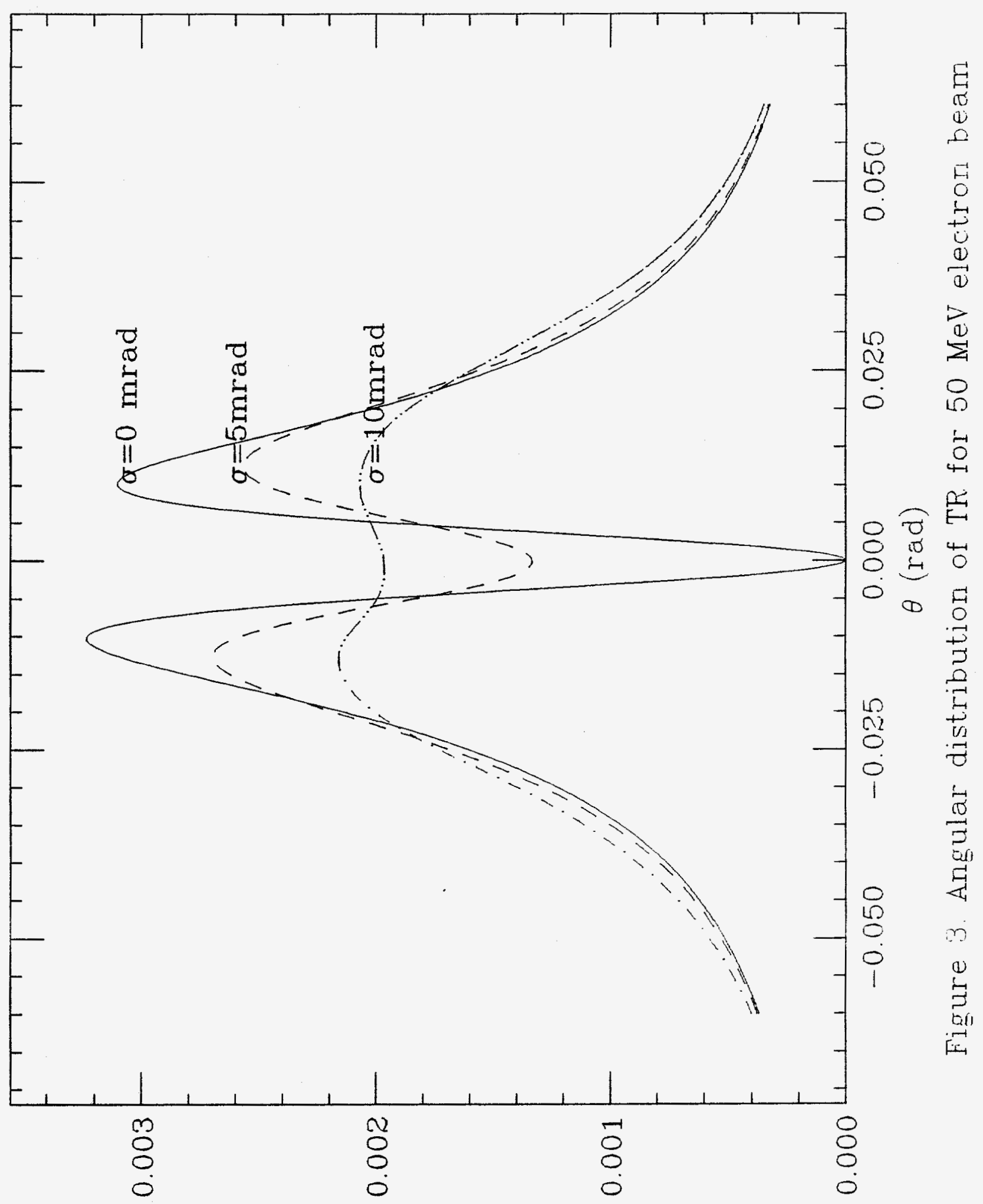

(Is/uru/suofoud) mp 


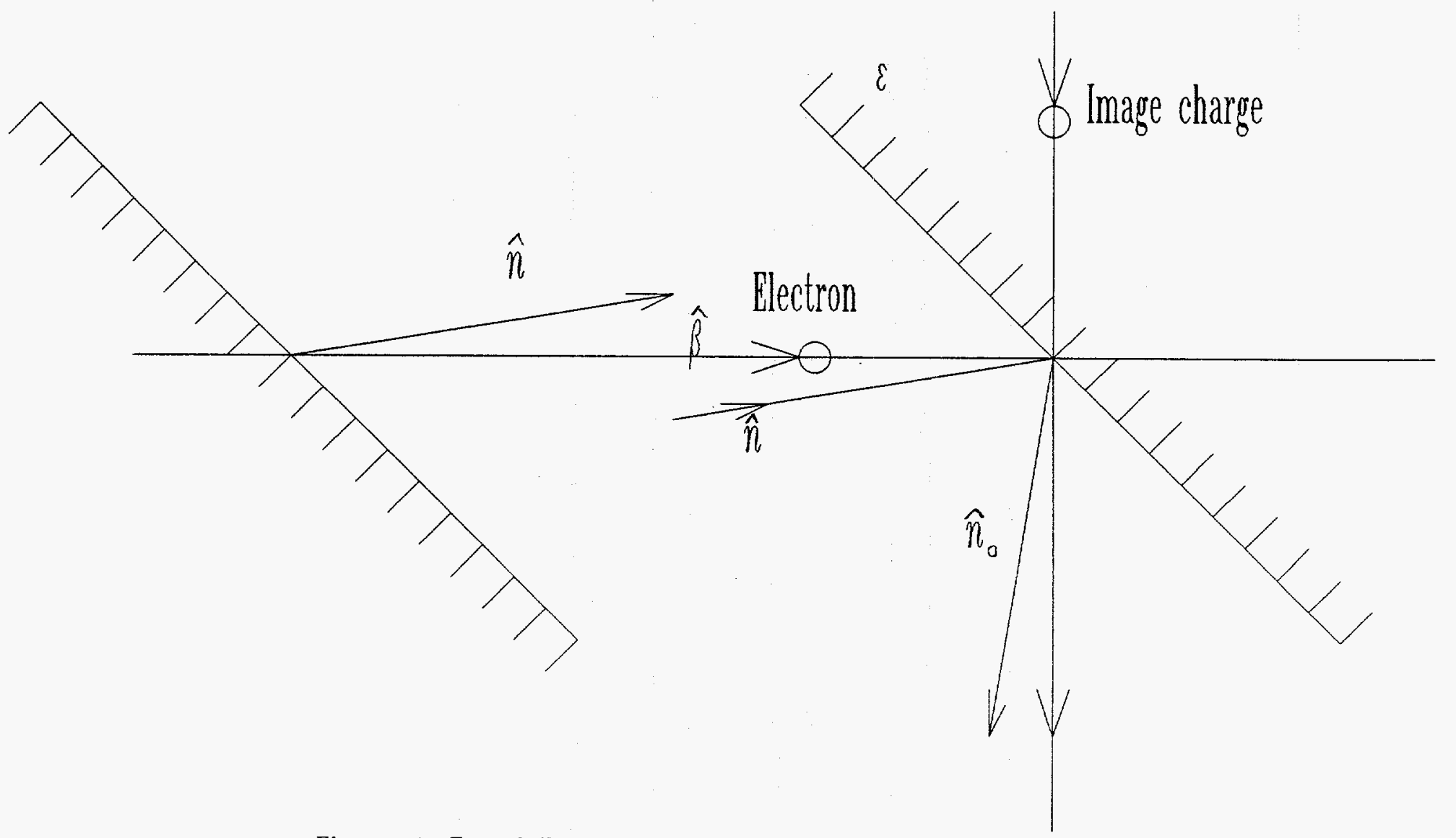

Figure 4. Two-foil transition radiation interferometer 


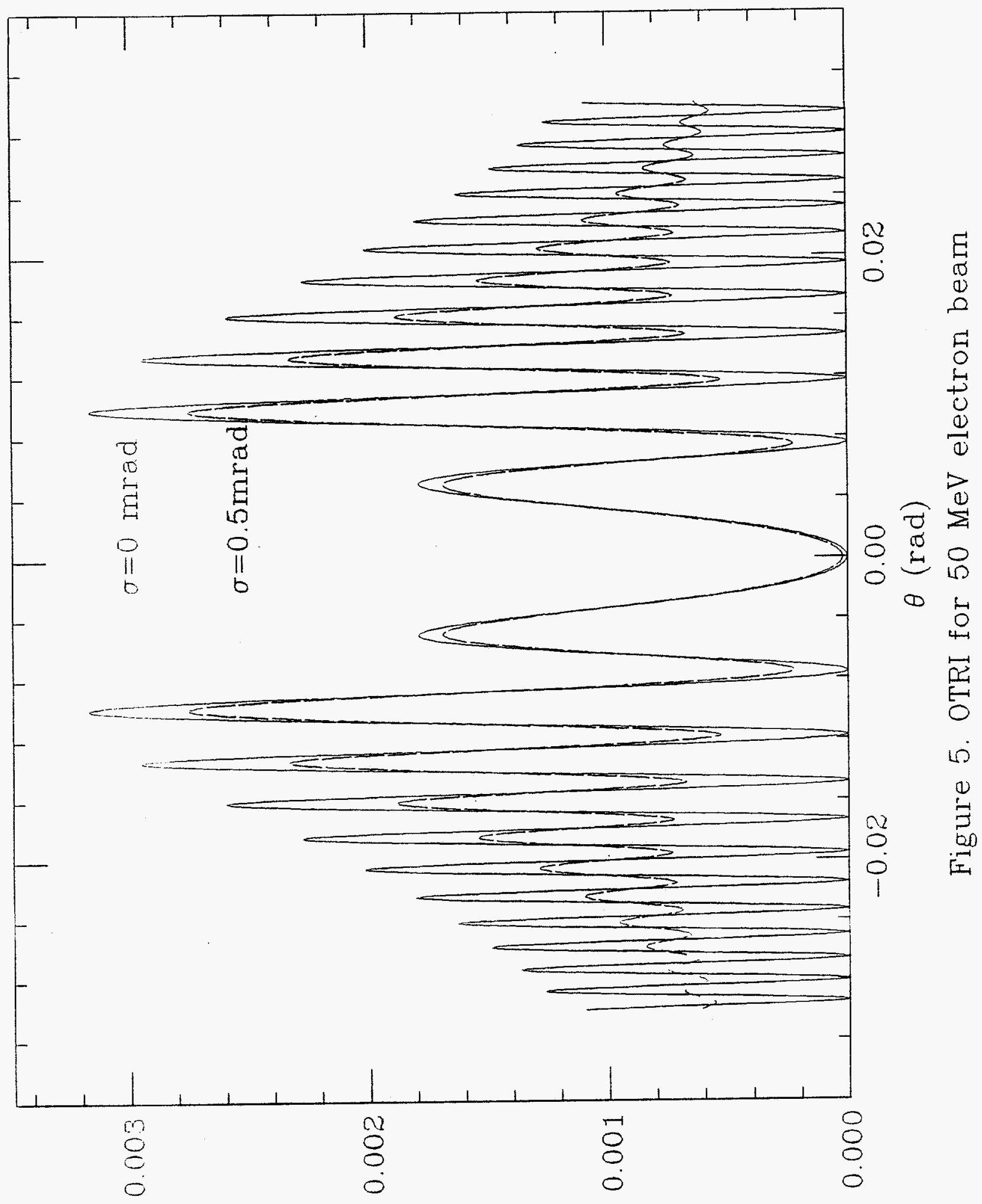

(IS/Uru/strozoud) mpVp/IP 


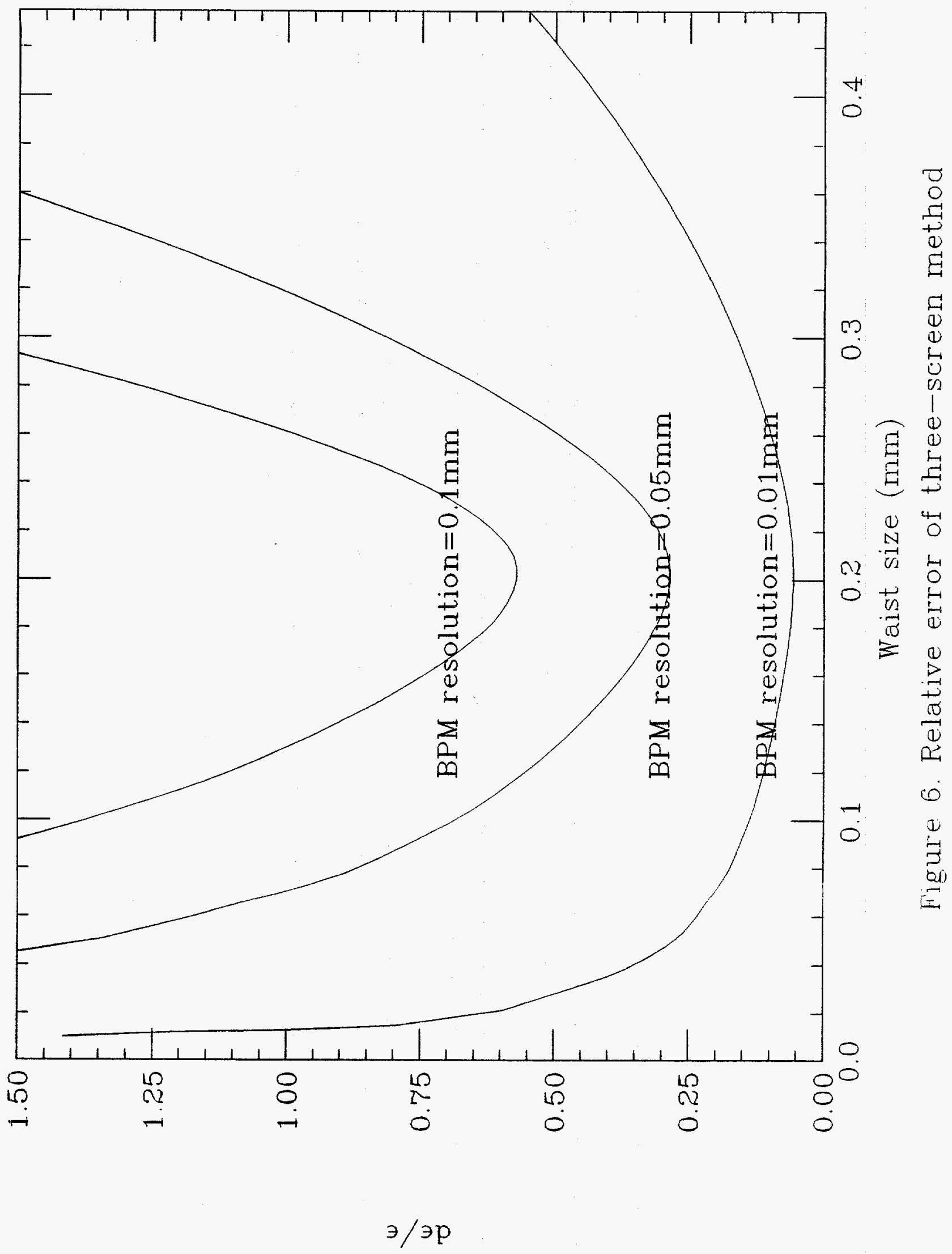




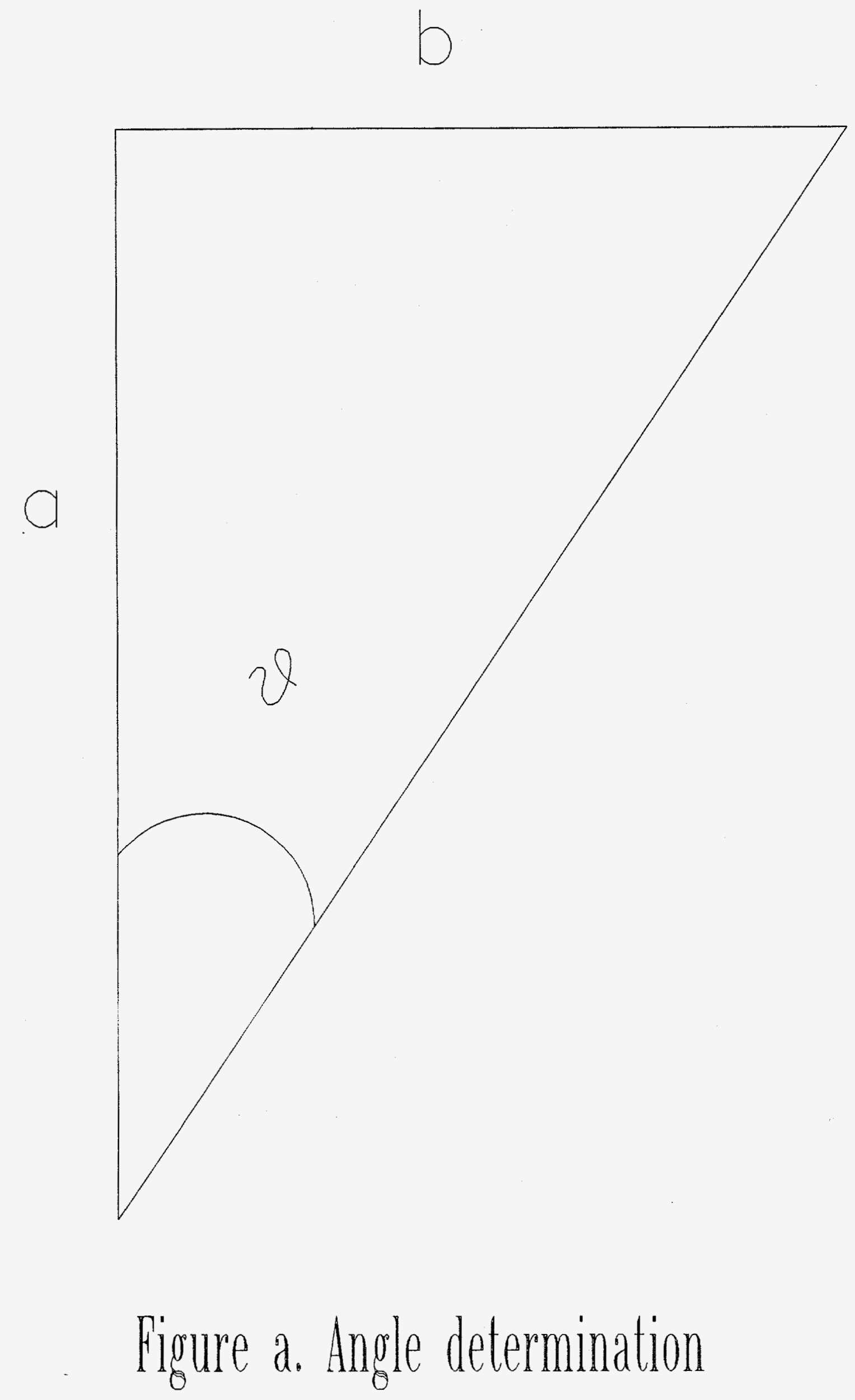

